\title{
LAS COOPERATIVAS EN EL DESARROLLO DEL ESPACIO RURAL. EXPERIENCIAS EN LA PROVINCIA DE MÁLAGA (ESPAÑA)
}

\author{
C. NIETO FIGUERAS \\ Departamento de Geografía. Universidad de Málaga. \\ Facultad de Filosofía y Letras, \\ Campus Teatinos s/n, 29071 Málaga \\ Correo electrónico de contacto: cristinanf@hotmail.com
}

\begin{abstract}
RESUMEN. La finalidad de este trabajo es mostrar la función que desempeñan las sociedades cooperativas en los procesos de desarrollo rural. Se analizan sus principales características así como las cualidades que las hacen especialmente idóneas para el impulso y el sostenimiento social y económico de determinados espacios. El caso de la provincia de Málaga permite confirmar su papel en la generación de empleo y en la integración laboral de colectivos desfavorecidos, como el femenino.
\end{abstract}

ABSTRACT. The objective of this work is to show the function that cooperatives play in rural development. Their main characteristics and means are analyzed to know the way these firms foment the development and encourage the economics and society of rural spaces. Some examples in Malaga are studied, and we can confirm their positive role in unemployment problem.

Palabras clave: Economía social, desarrollo rural, cohesión social, cooperativas, asociaciones.

Key words: Social economy, rural development, social cohesion, cooperatives, associations.

Enviado el 21 de marzo de 2005

Aceptado el 10 de junio de 2006

\section{Introducción}

La formulación del desarrollo rural actualmente en vigencia se basa en la superación del modelo de crecimiento agrario y en la generalización de presupuestos más amplios, donde la idea de lo rural responde a los diferentes significados de una sociedad 
más compleja, como resultado de un proceso de redefinición y una profunda transformación (Navarro y Larrubía, 2001).

Esta nueva sociedad precisa ser atendida desde un enfoque plurisectorial que sea capaz de afrontar sus necesidades y carencias. Tanto es así, que la reflexión científica sobre la realidad da como resultado el modelo de desarrollo rural endógeno, que emerge tras una evolución conceptual desde un desarrollo rural inicialmente dirigido al Tercer Mundo, hasta la definición de un modelo de desarrollo de componente local destinado a las áreas rurales desfavorecidas en el contexto de las sociedades postindustriales. Su formulación teórica y práctica se basa en la consideración y reconocimiento de los desequilibrios entre regiones y del carácter específico de los procesos económicos y sociales que inciden en la sociedad rural (Larrubia et al., 2004). La atonía que caracteriza a buena parte de estas áreas se traduce en tejidos productivos débiles, que no sólo desaprovechan las potencialidades del entorno sino que además se muestran incapaces de responder a las demandas de empleo de una población que, en consecuencia, opta cada vez más por la oferta exterior, circunstancia a menudo ligada a la despoblación (Ocaña, 1995).

En consonancia con lo anterior, el modelo de desarrollo rural endógeno se apoya en varios elementos: en primer lugar, la finalidad de crecimiento económico es sustituida por la de desarrollo social y económico, con la mirada puesta en el logro de cohesión e igualdad entre las áreas (Etxezarreta, 1988). En segundo lugar, el enfoque sectorial deja paso al integrado, entendiendo que el desarrollo debe atender todos los ámbitos y todos los sectores (Ceña Delgado, 1993). En tercer lugar, el éxito y el sostenimiento del desarrollo precisa de una serie de factores entre los cuales destacamos: el espacio receptor de los procesos, que deja de ser un mero receptáculo para convertirse en elemento activo del desarrollo; la nueva referencia territorial, que centra la atención en el marco local, aunque sin perder de vista la necesaria integración de éste en el más amplio contexto global; los recursos humanos como elemento esencial en los procesos, de acuerdo con el papel a desempeñar por la población autóctona en lo que debe ser un desarrollo endógeno; es fundamental la tecnología y su acercamiento a la población, acercamiento que consiste no sólo en la disponibilidad de aquélla sino en el conocimiento y óptimo aprovechamiento de la misma por parte de ésta; el papel de la intervención pública, que se caracteriza por la mayor responsabilidad de los gestores locales, y por la importancia concedida a la concertación y colaboración entre las instancias de todas las escalas competenciales (Vázquez Barquero, 1987).

Los proyectos en los que se espera descanse el desarrollo son de pequeña dimensión, frente al protagonismo que en momentos anteriores han tenido los de gran envergadura. De acuerdo con lo anterior y con la pretensión de implicar a la población local, las pequeñas y medianas empresas del entorno constituyen el prototipo de entidad (Vázquez Barquero, 1988). Los investigadores llegan a similares conclusiones respecto a la importancia de las PYMEs en el desarrollo: el tipo de empresas defendido por el modelo de desarrollo local son entidades de pequeño y mediano tamaño del entorno local, que se consideran uno de los ejes del desarrollo debido a que son intensivas en empleo de mano de obra, por lo que desempeñan un importante papel como creadoras de empleo, y extensi- 
vas en factor capital, de modo que no necesitan grandes inversiones para funcionar, elemento que las sitúa al alcance de economías más modestas sin que se precise necesariamente la adopción de riesgos financieros inabarcables para la población local. Además de ello, su pequeño tamaño y flexibilidad les proporciona mayor capacidad de adaptación a los cambios por el lado de la oferta y la demanda (Valcárcel-Resalt, 1999).

Así, la creación de microempresas y el autoempleo prometen unas perspectivas muy interesantes en el medio rural, de hecho aquéllas son interpretadas como un importante factor de renovación del tejido económico y el impulso a su creación y desarrollo va a ser uno de los objetivos de las estrategias de desarrollo endógeno.

Llegados a este punto, conviene señalar que el desarrollo endógeno no carece de valoraciones marcadas por la prudencia. Desde la perspectiva de este trabajo, interesa destacar el cuestionamiento planteado por la validez de todas las PYMEs como tipo ideal de empresa. Los investigadores advierten ante la excesiva confianza que se ha depositado en ellas, señalando en primer lugar que la capacidad de generar puestos de trabajo se opone a la viabilidad de este tipo de entidades en el libre mercado (Rego Veiga, 1992). Ante los niveles de competitividad que exigen los mecanismos e interrelaciones del sistema productivo capitalista, no parece posible la supervivencia de estas células empresariales sin estrategias de intervención estatal, salvo que se proceda a otro tipo de prácticas. En este sentido, la integración empresarial o las fórmulas de la Economía Social pueden entrar bajo la consideración de los técnicos y de las mismas empresas.

Según se desprende de lo expuesto, la Economía Social se presenta como la posible alternativa para afrontar algunos de los problemas ligados a las pequeñas o muy pequeñas empresas, y a sus dificultades para sobrevivir en el mercado en competencia con empresas mayores.

En este orden de cosas, el presente trabajo tiene dos líneas expositivas: la primera de ellas consiste en encuadrar los vínculos entre la Economía Social y el desarrollo rural. A ello dedicaremos el siguiente epígrafe.

La segunda línea se centra en el análisis del caso específico de la provincia de Málaga, en el marco de una investigación ligada a una tesis doctoral sobre el empresariado femenino (Nieto, 2003).

El estudio incluía a 75 iniciativas empresariales de las cuales 20 son entidades de la Economía Social, tratándose en concreto de 17 cooperativas y 3 sociedades laborales. Los objetivos del trabajo estuvieron encaminados, entre otros, a analizar el empresariado femenino rural desde la perspectiva del desarrollo rural. A este respecto la investigación se planteó con la intención de encuadrar las experiencias en el modelo de desarrollo, valorando el papel que están desempeñando en los procesos de reactivación económica de las áreas rurales de la provincia de Málaga, ámbito espacial de referencia, en reconocimiento a la dificultad que caracteriza a estos espacios para incorporarse al ritmo de crecimiento de su entorno, y a la función que en este sentido se le está atribuyendo, desde la teoría del desarrollo rural, a las iniciativas empresariales. 
Desde la perspectiva de este trabajo, además de lo expuesto, interesa prestar atención a otro de los objetivos de la tesis, desde el cual se contemplan las experiencias consideradas desde el contexto del mercado laboral, partiendo de la hipótesis posteriormente corroborada de que un marco laboral limitado y poco atractivo podía estar en el origen de muchas de las iniciativas empresariales, que venían a constituir la respuesta de las protagonistas, por la vía del autoempleo, a circunstancias tan adversas.

\section{Metodología}

Para cubrir los objetivos descritos se siguieron diferentes pautas metodológicas. El primer paso consistió en la exploración de las fuentes estadísticas y documentales, lo que supuso un considerable esfuerzo de recopilación, por la dispersión de algunos datos, o por el difícil acceso a otros. En ocasiones se tuvo que realizar un tratamiento secundario a la información, para poder adaptarla a las necesidades de la investigación (por el sistema empleado por los organismos competentes para obtener y procesar la información).

La mayor parte de la investigación descansó esencialmente en el trabajo de campo, debido a las limitaciones de las fuentes documentales y estadísticas disponibles. Esta dificultad proporcionó, sin embargo, la posibilidad de adaptar los instrumentos de conocimiento, la entrevista y la encuesta, a las necesidades concretas del estudio, lo que hizo posible a su vez alcanzar un conocimiento profundo sobre innumerables cuestiones y desde muy interesantes puntos de vista, lo que habría sido imposible de contar sólo con las fuentes oficiales. Además de ello, el empleo complementario de ambas permitió superar los inconvenientes de cada una: en la entrevista, la posibilidad de reconducir el proceso y profundizar en determinados temas de interés contrarrestó el carácter cerrado de la encuesta.

La recopilación de la información primaria necesitó de un trabajo previo de conocimiento de la población de empresarias rurales malagueñas, que no se pudo realizar de la manera deseada por el carácter confidencial de la fuente estadística (Censo de Actividades Económicas, donde la protección de datos personales impedía conocer el nombre y procedencia de los titulares, necesario para aislar al colectivo de mujeres empresarias). Se emplearon por ello fuentes alternativas, como la Federación Andaluza de Empresas Cooperativas de Trabajo Asociado y el Servicio Provincial de la Mujer.

El trabajo de campo tuvo una duración superior a los dos años pues, además del desplazamiento a distintos municipios, la celebración de las entrevistas hubo de adaptarse a la disponibilidad de tiempo de las protagonistas.

\section{3. Área de estudio}

La atención prestada al empresariado rural femenino se debe en parte al peso que los planteamientos de desarrollo rural están concediendo a las iniciativas empresariales en los procesos de desarrollo local. Estos planteamientos se apoyan en el reconocimien- 
to de la situación crítica de gran parte del medio rural, que entre otros aspectos se evidencia en la incidencia de la emigración selectiva (mujeres y jóvenes) en la regeneración demográfica. Para lograr la reducción del éxodo femenino es preciso crear oportunidades laborales, pues el mercado laboral rural no puede absorber a la población activa femenina, ni cubrir las expectativas de un colectivo femenino mejor formado que en momentos anteriores. Esta crisis responde, en gran medida, a la realidad rural malagueña, lo que nos lleva al marco territorial de la investigación.

Situada en el extremo meridional europeo (Fig. 1), la provincia de Málaga presenta un espacio rural caracterizado por los contrastes entre áreas litorales beneficiadas por el crecimiento económico ligado especialmente al turismo, y zonas interiores situadas al margen de estos procesos. Es precisamente en este espacio de contrastes donde se sitúa la aparición de empresas rurales femeninas.

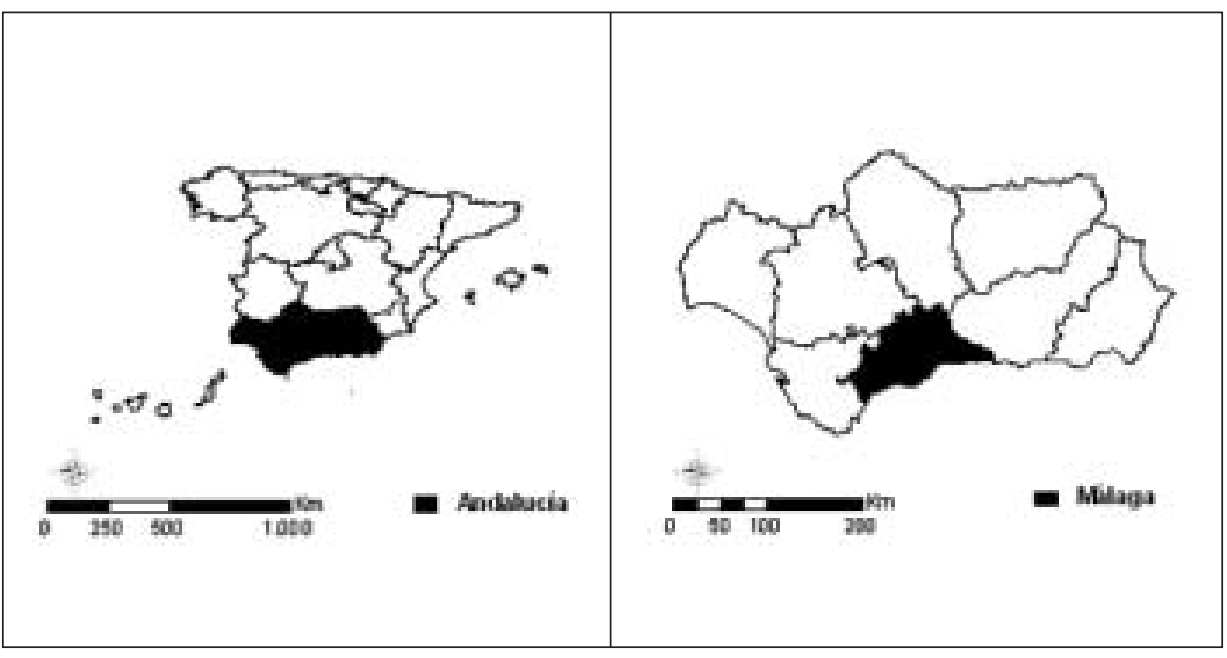

Figura 1. Área de estudio

\section{La Economía social y el desarrollo rural}

En este epígrafe realizaremos un breve acercamiento a sus principales características en orden a valorar hasta qué punto pueden responder a las carencias de las PYMEs. También veremos de qué manera las más altas esferas se hacen eco de este hecho, reconociendo la validez y conveniencia de promover el también llamado Tercer Sector.

\subsection{La Economía Social: las sociedades cooperativas}

La Economía Social integra a una serie de entidades de forma jurídica heterogénea, que se relacionan con todos los sectores de la actividad y con todas las etapas del proceso 
de producción. Se diferencian de las llamadas empresas de capital por medio de ciertas características comunes (Nieto, 2001). En primer lugar, la aplicación de los principios de solidaridad y responsabilidad constituyen unas de sus premisas, determinadas por la necesidad de generar beneficios al entorno y riqueza colectiva. En consonancia con esto, entre sus objetivos destaca la creación de empleo y la distribución equitativa de la riqueza.

En segundo lugar, la primacía del hombre sobre el capital determina que el trabajo y las personas que integran estas entidades tengan mayor peso que el capital en el reparto de beneficios. Si a ello se añade la superación de la dimensión puramente económica o, lo que es lo mismo, la finalidad meramente lucrativa como objetivo final, se evidencia claramente la diferencia de este sector frente a la economía privada capitalista. El hecho de que entre sus principios declaren que su gestión es autónoma e independiente de los poderes públicos, las sitúa en un plano diferente a la economía pública o estatal. Por ello también se les conoce como Tercer Sector, término genérico que las distingue de la economía privada y de la economía pública.

Como se ha expuesto con anterioridad, muchas y variadas son las organizaciones jurídicas que integran el sector (Buendía, 1999). De acuerdo con los objetivos de este trabajo, nos interesa hacer hincapié en las sociedades cooperativas, consideradas como el eje principal de la Economía Social.

Las cooperativas son organizaciones empresariales que forman parte de las llamadas empresas de participación, expresión que hace referencia a su doble vertiente empresarial de un lado, y democrática de otro. En ellas se asocian personas con intereses socioeconómicos comunes, que toman a la persona como elemento principal de su estructura organizativa y que, con total sometimiento a las reglas del mercado, tienen como objetivo responder a sus necesidades e intereses.

En un primer nivel se clasifican las cooperativas de primer y segundo grado. Las primeras constituyen las unidades básicas que pueden integrarse para dar paso a cooperativas de segundo y más grados. En un segundo nivel las cooperativas básicas o de primer grado se clasifican en cooperativas de trabajo asociado, de consumidores y usuarios, y cooperativas de servicios.

Dentro de la variedad de sociedades cooperativas que existen, las cooperativas de trabajo asociado son aquéllas que están integradas por personas físicas en calidad de socios, quienes realizan en común actividades económicas de producción de bienes y servicios destinados a terceros.

\subsection{Las cooperativas y el desarrollo rural}

Al principio de este trabajo se ha mencionado el papel que se le atribuye a las PYMEs en el modelo de desarrollo rural, así como las principales dudas suscitadas al respecto. En relación con la capacidad de las pequeñas y medianas empresas para mantenerse en el mercado sin el acompañamiento y la ayuda de los organismos públicos, 
cabe destacar los problemas de competitividad ligados a su pequeño tamaño. Se han señalado no pocas veces las dificultades financieras, fiscales y de otro tipo, que se encuentran relacionadas, entre otros aspectos, con la dimensión de la entidad. Además de sus demandas de flexibilización fiscal y otras medidas de acompañamiento de origen público, se plantean algunas alternativas que pueden contribuir a aligerar las cargas. Se trata de la integración en entidades mayores como pueden ser asociaciones, cooperativas o agrupaciones de empresas, a modo de plataforma para solventar ciertas carencias o para afrontar con la seguridad del grupo, cuestiones que en solitario se perciben como inabarcables. En efecto, este tipo de fórmulas permite cubrir determinados problemas financieros, de gestión, comercialización, formación, tecnología, etc. Un ejemplo de ello lo constituye la integración de cooperativas básicas en cooperativas mayores, llamadas de segundo o posterior grado, en las que la unión facilita el diseño y desarrollo de una estructura empresarial competitiva en el mercado, capaz de superar la precariedad en el trabajo.

Estas respuestas nos parecen las más deseables, en la medida en que esto suponga prescindir de la buena disposición e iniciativa ajenas y descansar, con mayor o menor dificultad, en la propia. Los autores reflexionan sobre la bondad de las cooperativas en este respecto, viniendo a coincidir en sentido positivo (García Marcos, 1999).

Pero su aportación al Desarrollo Rural no se restringe a su valor como respuesta a las limitaciones de las PYMEs. Para comprender mejor hasta qué punto las cooperativas constituyen un referente para el modelo de desarrollo, es necesario examinar brevemente los principios constitutivos de estas entidades. Los principios generales de constitución que dan base al funcionamiento de las cooperativas son los llamados principios cooperativos, que han quedado recogidos por la Alianza Cooperativa Internacional (ACI):

El primero es el principio de adhesión voluntaria y abierta, según el cual se trata de organizaciones de afiliación voluntaria y abiertas a todas las personas que deseen ser socias. El principio de gestión democrática señala que los socios participan activamente en la toma de decisiones, lo que en las cooperativas de primer grado se realiza de acuerdo con la norma un hombre un voto.

Según el principio de participación económica, los socios contribuyen equitativamente al capital de las cooperativas y lo gestionan de manera democrática. Por su parte, el principio de autonomía e independencia asegura que el control democrático de la cooperativa permanece en los socios así como la autonomía de la gestión en caso de acuerdo con terceros.

El principio de educación proporciona formación e información a los socios para mejorar su contribución al desarrollo de la sociedad y a la buena marcha de la propia cooperativa. El principio de cooperación entre cooperativas es una forma de fortalecer la entidad y su eficacia mediante la integración de ésta y otras en cooperativas mayores, asegurando el trabajo conjunto de las unidades de diferentes ámbitos espaciales. 
Por último, el principio de compromiso con la comunidad consiste en el interés por generar beneficios sociales y económicos en el entorno, con el fin último de promover el desarrollo sostenible.

Algunos de los principios que se han señalado apuntan claramente hacia los planteamientos de desarrollo rural. El compromiso con el desarrollo del entorno implica una serie de pautas de comportamiento que han podido constatarse en las experiencias analizadas en este estudio. Se trata entre otros del interés en generar beneficios en el entorno inmediato, así como del objetivo de crear empleo como fin social.

La dimensión social de estas empresas no sólo se pone de manifiesto de esta manera, ya que constituyen un tejido social en la medida en que la persona tiene mucho más peso que el beneficio económico. Del mismo modo, estas entidades contribuyen a un crecimiento sostenido que se apoya, entre otros elementos, en la implicación real de los socios en tanto que su intervención en la gestión es directa y personal, lo que garantiza su participación y compromiso en cada proyecto empresarial. Por último, hay que señalar que el interés por el desarrollo sostenible (último de los principios citados) remite directamente al compromiso con el entorno y el medio ambiente.

Lo que se ha expuesto con anterioridad se identifica sin duda con los principales factores de éxito del modelo de desarrollo rural: implicar a la población local; potenciar los recursos humanos como elemento activo de los procesos; generación de empleo (Larrubia et al., 2004).

Al primero de los factores mencionados ya hemos hecho referencia anteriormente, a través del grado de implicación de la población local mediante la participación de los socios.

Por lo que respecta a los recursos humanos, éstos desempeñan un papel creciente en los planteamientos de desarrollo desde la consideración de que éste ha de ser autocentrado y, en consecuencia con ello, la población autóctona debe formar parte activa en los procesos. Este presupuesto teórico se hace una realidad en las cooperativas, no sólo a través del ya mencionado compromiso de los socios en la empresa sino, muy especialmente, por medio del objetivo de crear empleo como uno de los fines sociales de mayor peso. En este orden de cosas, el principio de formación de los socios también contribuye a la potenciación de los recursos humanos, en la medida en que el capital humano se mide por los parámetros formativos, de modo que la educación y la cultura constituyen su valor añadido, como medio que facilita los procesos de desarrollo y como fin mismo de éstos.

En este sentido, la formación constante que las cooperativas proporcionan a los socios en cuestiones no necesariamente limitadas al trabajo o a la actividad llevada a cabo por la empresa, no sólo contribuye a la adquisición de aptitudes y actitudes y al enriquecimiento profesional y humano de los miembros. Además de ello permite superar las carencias del actual sistema de educación, señaladas por otras aportaciones (García Marcos, 1999).

La relación entre las sociedades cooperativas y el empleo es una cuestión que, dada su profundidad e interés, merece una atención específica. 


\subsection{Las cooperativas y la creación de empleo. El reconocimiento de los poderes públicos}

La vinculación de la Economía Social con el empleo se remonta al siglo XIX, en las primeras asociaciones obreras integradas por grupos de trabajadores en Europa Occidental, surgidas en respuesta al proceso de mecanización extendido con la Revolución Industrial. Desde las primeras experiencias se ha evidenciado su estrecha relación con lo local y su identificación con los principios de solidaridad, democracia y responsabilidad.

Como se ha expuesto anteriormente, uno de los principios cooperativos es la generación de beneficios económicos y sociales en el entorno. Esto hace de las cooperativas y las entidades de la Economía Social, en general, el marco idóneo para el diseño y aplicación de programas de desarrollo y de impulso al empleo, especialmente en los destinados a dinamizar áreas rurales deprimidas, que se ven beneficiadas no sólo por la dimensión social implícita, sino también por los efectos directos en términos de creación de empleo y la consecuente fijación de la población al medio frente al éxodo rural.

Los investigadores han constatado la incidencia positiva en el empleo de estas entidades. En el ámbito europeo, se ha examinado la evolución de la Economía Social en todos los estados miembros centrando la atención en la trayectoria del empleo generado por estas empresas, en términos cuantitativos y cualitativos (VV.AA., 2000). Las conclusiones señalan que la mayor demanda de mano de obra por parte de la Economía Social se concentra en pequeñas entidades del sector servicios, de sectores ligados a la experimentación, y en general en empresas dedicadas a actividades de nuevo cuño, relacionadas con las nuevas necesidades de la sociedad occidental, y casi siempre por medio de experiencias centradas en el ámbito local. En el estudio destaca así mismo que el mayor dinamismo de estas empresas se encuentra íntimamente relacionado con determinadas actitudes por parte de las políticas públicas, encaminadas a prestar una atención especial a la promoción de la Economía Social y del empleo. Tanto es así, que las trayectorias y las situaciones actuales que mejor panorama presentan, se dan en los países con mayor tradición en este sentido, y que cuentan con amplios dispositivos y medidas públicas de apoyo.

Del mismo modo, las diferentes aportaciones dan cuenta de la toma de conciencia de las distintas esferas públicas respecto a la validez de estas empresas. Así, el Comité Económico y Social europeo reconoce en su dictamen del 26 de enero de 1990 la importante contribución que las empresas de la Economía Social hacen para el logro de la equidad social, en la misma línea en la que se sitúa el informe de la Comisión a la CE 'La Estrategia Europea en favor del Empleo' (García Marcos, 1996).

La Cumbre de Luxemburgo sobre el empleo supone un definitivo impulso a la Economía Social en tanto que en el Pilar II se vincula explícitamente a este tipo de empresas con las nuevas oportunidades de empleo y con el desarrollo de estas actividades en el ámbito local. En el texto del Pilar II, bajo el título 'Desarrollar el espíritu de empresa' y en el apartado 'Aprovechar las oportunidades de creación de puestos de trabajo', se afirma que la Unión Europea necesita 'aprovechar plenamente las posibilidades que ofrece la creación de puestos de trabajo a nivel local, en la economía social y en las nuevas actividades' como vía para luchar contra el problema estructural del desempleo. 
Ya desde la Cumbre de Lisboa se produce un significativo avance en las consideraciones respecto a la cohesión social, señalada como elemento que necesariamente ha de acompañar al desarrollo económico. La meta del pleno empleo se considera desde entonces como un instrumento necesario para la cohesión social. La consecución de la equidad social en paralelo al desarrollo económico demanda la colaboración entre los poderes públicos y los actores socioeconómicos, entendiéndose que la Unión y los Estados miembros deben implicar a la sociedad organizada. En este orden de cosas, la Economía Social se configura como pionera.

A ello se añade el papel de las distintas Conferencias Europeas de Economía Social, auténticos foros de reflexión y encuentro entre los representantes de las distintas Administraciones de los Estados miembros y de las organizaciones del sector de la Economía Social. Su celebración ha propiciado la creación de un cuerpo de doctrinas sobre la política a desarrollar en Economía Social. La III Conferencia celebrada en Salamanca en 2002, ha puesto el acento en la valoración de la función del sector en la cohesión de la sociedad de manera más equilibrada, a través de su acción para la integración social de las personas. Se ha hecho hincapié, entre otras cosas, en la respuesta que la Economía Social ha dado al problema del empleo, generando puestos de trabajo más estables y de mejor calidad que los del sistema económico, contribuyendo a paliar el éxodo rural; al fomento de la capacidad emprendedora y empresarial; a la cohesión e inserción laboral de personas o colectivos en riesgo de exclusión por sus especiales dificultades; a las nuevas necesidades y demandas sociales, a través de actividades que cubren servicios insuficientemente atendidos por la protección social, etc.

La IV Conferencia celebrada en Praga en octubre de 2002 continúa en la misma línea, aunque desde la perspectiva de este trabajo conviene destacar las conclusiones de un seminario sobre 'Desarrollo Local Sostenible y la asociación entre autoridades locales y regionales y la Economía Social', celebrado en el marco de esta Conferencia. Organizado por el Comité de las Regiones en cooperación con REVES (Red europea de ciudades y regiones para la Economía Social), contó con la participación de representantes de los gobiernos locales y regionales, y de las organizaciones de Economía Social de la Unión Europea y países candidatos. Entre las conclusiones alcanzadas, cobra especial interés la declaración en la que se reclama la adopción de medidas encaminadas a ampliar y mejorar las asociaciones entre gobiernos locales y el sector. Del mismo modo, se insta a la Unión Europea a la creación de una base de datos para la Economía Social y el desarrollo local y regional, y a la mayor flexibilización en la financiación de las asociaciones de la Economía Social a través de los Fondos Estructurales y la próxima convocatoria del programa EQUAL.

Por otro lado, los representantes del Comité de las Regiones identifican el aumento de la red de asociaciones del sector como vía hacia una mayor cohesión y prosperidad en tanto que son instrumentos para mejorar los resultados y fomentar el espíritu de empresa. Señalan así mismo que estas asociaciones pueden desempeñar una importante función en el desarrollo social y económico, ya que estas entidades logran que los ciudadanos se comprometan a mejorar el desarrollo local. 
Los Estados miembros y la Comisión Europea dan un paso definitivo mediante la promoción de un texto consensuado de Reglamento de la Sociedad Cooperativa Europea. El objetivo del mismo es 'facilitar el desarrollo de las actividades transnacionales de las cooperativas, dotándolas de instrumentos jurídicos adecuados que tengan en cuenta sus características específicas. Permitir la creación de nuevas cooperativas de personas físicas y jurídicas a escala europea. Garantizar los derechos a la información, consulta y participación de los trabajadores en una sociedad cooperativa europea' (Diario Oficial, 2003).

En un nivel más cercano, la Comunidad Autónoma Andaluza se hace eco de los planteamientos expuestos a través del diseño y aplicación de una serie de programas de fomento a las empresas y al autoempleo mediante entidades de la Economía Social, articulados de forma coordinada con la Administración Central y con la Unión Europea. Estos programas actúan promocionando directamente la creación de empleo a través de medidas como incentivos, subvenciones o avales a inversiones o proyectos de Economía Social que incidan positivamente en la inserción laboral. De las medidas de fomento, desagregadas en doce programas, recogemos algunas tan significativas como las subvenciones a fondo perdido a la inversión en proyectos que generen o estabilicen el empleo; la concesión de avales para lograr financiación externa; la reducción de los intereses en préstamos de hasta $3000 €$ por socio trabajador; las subvenciones al empleo en cooperativas, de hasta $3000 €$ por cada nuevo socio; el Programa Escuela de Empresas, destinado a fomentar iniciativas empresariales desarrolladas por jóvenes por medio de subvenciones para la construcción y funcionamiento de la escuela, etc.

La aplicación y desarrollo de estas medidas se ha dejado sentir en la trayectoria de las empresas de Economía Social en Andalucía, habida cuenta que su puesta en práctica ha coincidido con un fortalecimiento del ritmo de crecimiento del sector en los últimos años, lo que hace esperar un efecto similar en el empleo ligado al mismo.

\section{Resultados en la provincia de Málaga}

En las páginas que siguen atenderemos la situación de las empresas del sector (Economía Social), a partir de una serie de elementos muy relacionados con este trabajo. Lo que se pretende con ello es reflexionar en torno a dos cuestiones fundamentalmente. En primer lugar, en qué medida se integran estas experiencias en la economía de sus entornos locales o, lo que es lo mismo, hasta qué punto cumplen con el papel que se les presupone desde el modelo de desarrollo vigente. En segundo lugar y en directa relación con lo anterior, conocer si la reconocida incidencia de las empresas de la Economía Social en la generación de empleo, es un hecho en el ámbito espacial y en los casos estudiados.

Como se mencionó páginas atrás, las cooperativas y sociedades laborales de la muestra constituyen un total de 20, cuya relación figura en la tabla 1. Para ordenar las empresas se procedió a una primera clasificación por comarcas y, dentro de cada espacio comarcal, se ordenaron alfabéticamente a partir del municipio de localización. La codifi- 


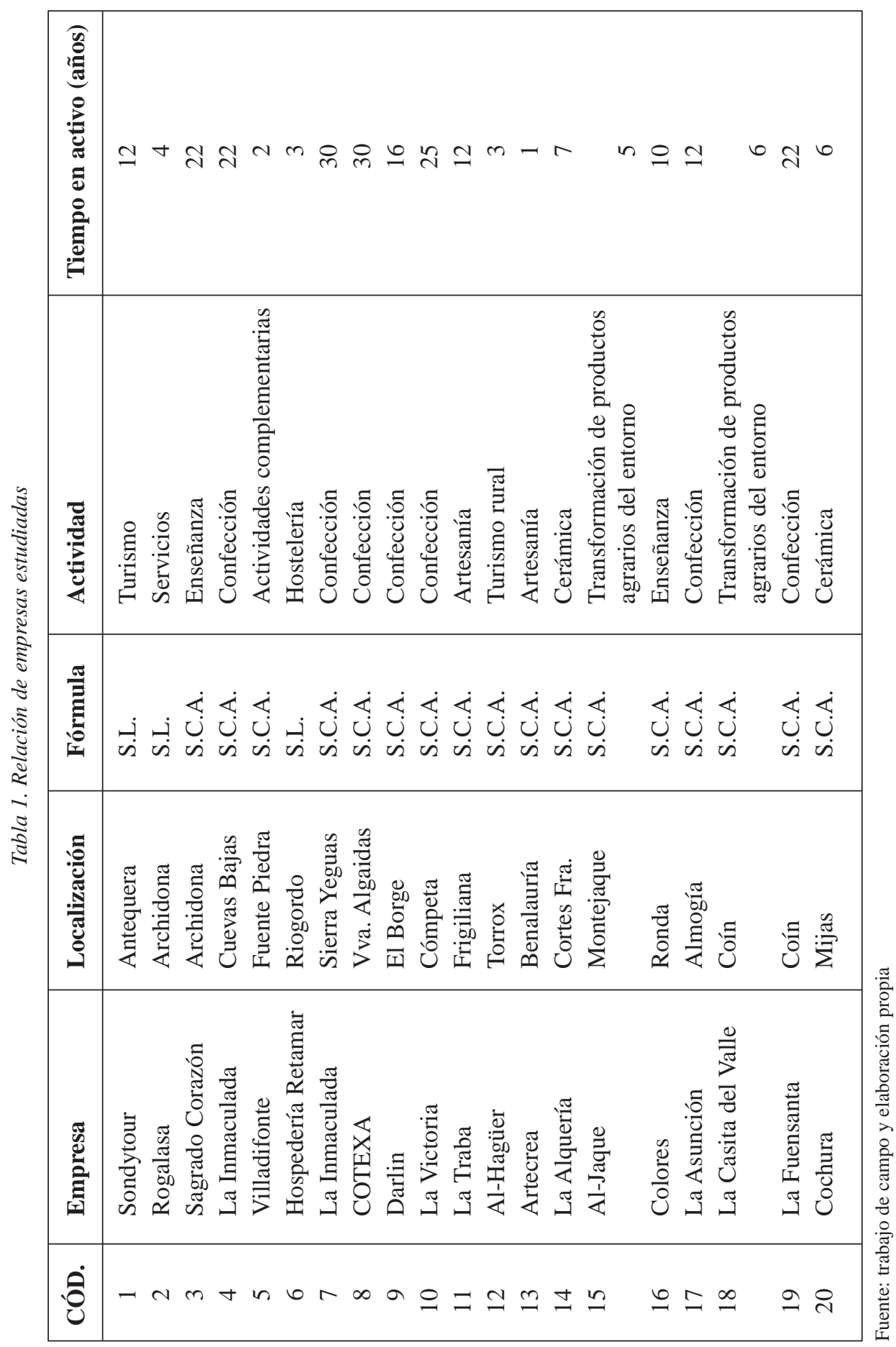


cación asignada a cada caso permite sintetizar la presentación en las restantes tablas. La información que da base a las tablas se ha extraído de las entrevistas y encuestas realizadas a las personas al frente de las entidades, siendo sintetizada y tabulada para facilitar tanto el análisis como la posterior exposición de los resultados. El primero de ellos presenta además la actividad desarrollada por la empresa así como el tiempo en activo en el mercado expresado en años desde su constitución.

Los datos permiten alcanzar algunas conclusiones. La distribución sectorial muestra la clara tendencia de las empresas hacia actividades encuadradas entre las llamadas nuevas oportunidades de empleo (Carrillo, 1999), excepto las cooperativas textiles, que se desmarcan de las líneas generales no sólo por la dedicación sino por otros muchos aspectos en los que no vamos a profundizar en este trabajo por apartarse de los objetivos del mismo. Tan sólo apuntar brevemente que la aparición del cooperativismo textil en los setenta del pasado siglo respondió a una situación de desempleo y de emigración, en un contexto laboral caracterizado por el predominio del sector primario y por el desempleo. A ello se unió la necesidad de mano de obra barata y flexible por parte de las grandes empresas confeccionistas, dando lugar a la emergencia de este tipo de empresas. En su momento trajo consigo mejoras cualitativas y cuantitativas en la vida familiar, en la medida en que el trabajo de las mujeres en las cooperativas proporcionó ingresos estables en un contexto socioeconómico difícil. En la actualidad atraviesan una profunda crisis relacionada con su dependencia de las grandes industrias textiles y con las condiciones del mercado internacional de trabajo, marcadas por la competencia de mano de obra barata de otros países o de la economía informal del trabajo a domicilio. A pesar de ello, continúan ejerciendo un peso muy importante en la generación de empleo y las perspectivas del sector parecen mejorar de la mano de ciertas reacciones observadas en buena parte de las experiencias entrevistadas, como es el caso de la integración en cooperativas de segundo grado (se trata de Málaga Cinco, integrada por las cooperativas codificadas con los números 7 y 8 , además de otras 3 no incluidas en el estudio), o de la reconversión del sistema productivo y el cambio de producto (caso de la cooperativa $n^{\circ} 4$ ).

Este trabajo ha permitido confirmar las altas expectativas generadas por la Economía Social. Ello es así por la vinculación de las experiencias analizadas con las actividades que cubren las necesidades sociales insuficientemente atendidas por el sector público, como los servicios a domicilio (experiencia $\mathrm{n}^{\circ}$ 2) o la enseñanza de calidad (experiencias $n^{0} 3$ y 16), así como con otras prácticas también integradas en las nuevas oportunidades de empleo, como las relacionadas con el turismo y actividades complementarias (experiencias $\mathrm{n}^{\circ} 1,5,12$ ), con la explotación de recursos locales (experiencias $\mathrm{n}^{\circ} 15$ y 18), o con actividades basadas en la pervivencia de tradiciones autóctonas (experiencias $\mathrm{n}^{\mathrm{o}} 11,13$ y 20).

La tabla 2 recoge diferentes cuestiones que contribuyen a definir la posición de las empresas en el marco del desarrollo rural, de acuerdo con los planteamientos que se han expuesto en la primera parte de este trabajo. Así, los aspectos que componen lo que hemos integrado bajo el epígrafe 'Relaciones con el entorno' constituyen la expresión de algunos de los factores de éxito del modelo. Considerando el carácter biyectivo de las 
Tabla 2. Algunos aspectos de la relación entre las empresas y el desarrollo rural

\begin{tabular}{|c|c|c|c|c|c|c|}
\hline \multirow[b]{2}{*}{ CÓD. } & \multicolumn{3}{|c|}{ Infraestructura y tecnología } & \multicolumn{3}{|c|}{ Relaciones con el entorno } \\
\hline & $\begin{array}{l}\text { Nivel } \\
\text { actual }\end{array}$ & $\begin{array}{l}\text { Planes } \\
\text { futuros }\end{array}$ & $\begin{array}{l}\text { Acceso a } \\
\text { Internet }\end{array}$ & Plantilla & Otras & Medidas de apoyo \\
\hline 1 & 1 & $*$ & $*$ & 2 & $*$ & SE \\
\hline 2 & 1 & $*$ & & 9 & $*$ & SE-AT \\
\hline 3 & 2 & $*$ & $*$ & 7 & $*$ & \\
\hline 4 & 1 & $*$ & $*$ & 30 & $*$ & SE-SI \\
\hline 5 & 3 & $*$ & $*$ & 2 & $*$ & SE-AT \\
\hline 6 & 2 & $*$ & $*$ & 13 & $*$ & $\mathrm{SE}$ \\
\hline 7 & 1 & $*$ & & 56 & $*$ & SE-SI \\
\hline 8 & 1 & * & & 82 & $*$ & SE-SI \\
\hline 9 & 3 & & & 14 & $*$ & SE-SI \\
\hline 10 & 3 & & & 5 & & SE \\
\hline 11 & 2 & $*$ & $*$ & 5 & $*$ & SE-SI-PL \\
\hline 12 & 2 & & $*$ & 1 & $*$ & SE-AT-PL \\
\hline 13 & 3 & $*$ & $*$ & 3 & $*$ & SE-AT \\
\hline 14 & 2 & $*$ & & 4 & $*$ & SE-SI-AT \\
\hline 15 & 2 & & $*$ & 3 & $*$ & SE-SI-AT-PL \\
\hline 16 & 2 & & $*$ & 6 & $*$ & SE-AT-EE \\
\hline 17 & 2 & $*$ & & 29 & $*$ & SE-SI \\
\hline 18 & 2 & $*$ & $*$ & 3 & $*$ & SE-SI-EE-PL \\
\hline 19 & 1 & $*$ & $*$ & 100 & $*$ & SE-SI \\
\hline 20 & 1 & $*$ & & 4 & $*$ & SE-SI-AT \\
\hline
\end{tabular}

Nivel infraestructura

1: Bueno

2: Medio

3: Bajo

*: Respuesta afirmativa

Fuente: trabajo de campo

Elaboración propia
AT: Asistencia técnica

SI: Subvenciones a la inversión

EE: Escuela de Empresas

SE: Subvenciones al empleo

PL: Proyectos locales de economía social 
relaciones entre la empresa y el entorno, de un lado se recogen las incidencias positivas de las experiencias en sus contextos locales, donde al empleo generado se unen aportaciones agrupadas bajo la expresión 'otras': consumo de servicios y/o materias primas del entorno, actividades concertadas y consumo indirecto a través de los clientes. De otro lado, las subvenciones y medidas de apoyo procedentes de organismos de distintas escalas competenciales se identifican con otro de los factores de éxito, el papel de la Administración y de los agentes locales. En el caso de la información obtenida a partir de entrevistas, nos hemos limitado a recoger las referencias relacionadas con las distintas medidas e instrumentos de fomento a la Economía Social, tratadas en páginas anteriores, cuya financiación procede mayoritariamente de fondos europeos (FEDER, FSE), gestionados y canalizados a través de los organismos públicos regionales y locales, que ocasionalmente colaboran con la aportación de capital.

Las relaciones con el entorno muestran que la incidencia positiva de las empresas es real y efectiva, habida cuenta que todas generan al menos uno de los beneficios considerados (empleo y otras); el empleo generado es estable en su totalidad, además el volumen total de las plantillas coincide también con lo apuntado por las referencias europeas recogidas, pues denota la mejor capacidad empleadora de estas entidades en relación con el resto del estudio, siendo que las 20 empresas representan tan sólo el 26\% de la muestra original, mientras que concentran a más del 66\% del empleo total.

El grado de compromiso de las protagonistas con el desarrollo de sus entornos y el papel de estas empresas ante el problema del empleo se evidencia especialmente en las motivaciones que animaron las iniciativas. En este aspecto los testimonios no dejan lugar a dudas respecto al peso que las escasas oportunidades laborales adquieren en la decisión de emprender. Del mismo modo, otra de las cuestiones que animaron la emergencia de buena parte de las entrevistadas fue la voluntad de crear empleo y beneficios colaterales (experiencias $n^{\circ} 2,5,15$ ), así como el deseo de aprovechar el potencial ligado a los recursos locales (experiencias $n^{\circ} 5,11,15,18$ ), todo ello en clara sintonía con los argumentos del modelo de desarrollo.

Las medidas de apoyo han tenido un peso muy importante en las iniciativas empresariales. Es posible afirmar que de modo genérico se han visto cumplidos los objetivos de las políticas de fomento a la Economía Social, que recordemos estaban encaminados a promover la generación de empleo y el espíritu emprendedor. Nuestras experiencias así lo muestran, no sólo por la presencia de medidas en la práctica totalidad de los casos. Algunos de los testimonios reconocen que la percepción de subvenciones fue esencial en el inicio de la actividad, tanto es así que sin este componente habrían visto seriamente comprometida la iniciativa (experiencias $\mathrm{n}^{\mathrm{o}} 1,2,5,12,15,18$ ).

En cuanto al tipo de medidas, las de tipo económico no son las más aplaudidas pese a su evidente importancia, siendo que la asistencia y apoyo en cuestiones técnicas y en la gestión de los trámites burocráticos constituye para muchas un factor imprescindible, especialmente en los primeros y más difíciles tiempos. Así pues, se advierte el papel de los poderes públicos (de diversas escalas competenciales) como factor de éxito del modelo de desarrollo, al menos en lo que a los casos analizados se refiere. 
Tabla 3. Crecimiento y perspectivas de las empresas

\begin{tabular}{|c|c|c|c|c|c|c|}
\hline \multirow{2}{*}{ CóD. } & \multicolumn{2}{|c|}{ Facturación } & \multirow{2}{*}{$\begin{array}{l}\text { Planes } \\
\text { futuros }\end{array}$} & \multicolumn{3}{|c|}{ Procedencia del capital } \\
\hline & Actual & Evolución & & Autofinanciación & Subvenciones & Créditos \\
\hline 1 & 5 & + & I-C-L-R-T & $*$ & & $*$ \\
\hline 2 & 3 & + & I-C-D-L-T & & $*$ & $*$ \\
\hline 3 & & & I-C-L-R-T & & $*$ & $*$ \\
\hline 4 & 5 & + & I & $*$ & $*$ & $*$ \\
\hline 5 & 2 & + & I-C-D-R & & $*$ & $*$ \\
\hline 6 & 4 & + & I-D & $*$ & $*$ & $*$ \\
\hline 7 & 6 & + & I & $*$ & $*$ & $*$ \\
\hline 8 & 6 & + & $\mathrm{I}-\mathrm{L}$ & $*$ & $*$ & $*$ \\
\hline 9 & 3 & $=$ & $\mathrm{L}$ & $*$ & $*$ & \\
\hline 10 & 2 & - & & & & \\
\hline 11 & 2 & + & I-D & $*$ & * & $*$ \\
\hline 12 & & + & I-D-P & $*$ & $*$ & $*$ \\
\hline 13 & & - & $\mathrm{L}$ & $*$ & $*$ & \\
\hline 14 & & + & I-P-R & $*$ & * & $*$ \\
\hline 15 & 3 & + & C-T & $*$ & $*$ & $*$ \\
\hline 16 & & & $\mathrm{~L}$ & & $*$ & $*$ \\
\hline 17 & 4 & $=$ & I & $*$ & $*$ & \\
\hline 18 & & + & I-D-P-R & $*$ & $*$ & $*$ \\
\hline 19 & 6 & + & I & $*$ & $*$ & $*$ \\
\hline 20 & & + & $\mathrm{I}-\mathrm{C}$ & $*$ & $*$ & \\
\hline
\end{tabular}

Facturación (miles de €)

$1:<6$

2: $6-30$

3: $30-90$

4: $90-180$

5: $180-600$

6: $>600$

*: Respuesta afirmativa

Fuente: trabajo de campo

Elaboración propia
Planes futuros

I: Infraestructura

L: Local

C: Incrementar la clientela

$\mathrm{T}$ : Incrementar la plantilla

D: Diversificación

R: Radio de acción 
Por lo que respecta a la infraestructura y tecnología, se trata también de uno de los factores básicos del modelo de desarrollo, por este motivo se hizo especial hincapié en conocer este aspecto de las empresas, en qué medida hacen uso de los avances tecnológicos (expresado en la columna 'nivel') y cuál es la actitud o la disposición frente a la incorporación de novedades (columna 'planes futuros'). Más por las intenciones inmediatas que por la situación actual, las perspectivas son bastante alentadoras.

La tabla 3 centra su atención en la proyección futura de las empresas, indagando sobre temas como los proyectos inmediatos o a medio plazo que las protagonistas están dispuestas a emprender, la procedencia del capital con el que se van a financiar, lo que a su vez permite conocer la disposición a asumir riesgos según la presencia de los créditos entre las respuestas así como acercarnos a la productividad de las iniciativas en función de la capacidad de autofinanciarse. El conocimiento de este aspecto es completado tomando en consideración la facturación del último año y la trayectoria descrita.

Todas estas cuestiones interesan en orden a valorar el grado de compromiso de la empresaria con su negocio y con el sostenimiento del mismo, y no sólo por su incidencia de cara a los procesos de desarrollo local. También centran la atención de este estudio en atención a valoraciones que se cuestionan el auge emprendedor, que relacionan la emergencia de empresas con la captación de recursos europeos temiendo que su permanencia en la actividad esté supeditada a la percepción de ayudas.

La amplia respuesta que los testimonios han dado a los planes de futuro, así como la generalizada actitud favorable al riesgo en los créditos, denotan la implicación de las protagonistas con sus empresas y la firme intención de no estancarse. Por otro lado, la capacidad de autofinanciar los proyectos, extensiva a la mayor parte de los casos, y la evolución de la facturación en los últimos años apuntan hacia una productividad y una prosperidad que permiten esperar el sostenimiento de las actividades en el mercado. En este sentido conviene destacar que la buena disposición al riesgo, corrobora lo que se ha dicho en ocasiones anteriores respecto a las ventajas del cooperativismo. Esto es especialmente evidenciado en las cooperativas textiles, en las que la integración en una cooperativa de segundo grado o simplemente el capital social (aportado por cada socio) permite que la autofinanciación sea una realidad.

\section{Conclusiones}

Tomando en consideración lo que se ha expuesto en la primera parte de este trabajo sobre el papel de la Economía Social en el desarrollo rural, y en lo relativo al reconocimiento de este hecho por parte de las instancias públicas, el análisis de las experiencias permite extraer una serie de conclusiones.

Las empresas analizadas confirman las bondades atribuidas a las PYMEs a la vez que responden a las limitaciones de éstas. Así, se ha constatado su mayor capacidad como fuente de empleo por su flexibilidad para adaptarse a circunstancias económicas difíciles 
y por responder más rápidamente a las nuevas oportunidades. Muestra de su adaptación a los cambios de la demanda es su capacidad de reciclarse en nuevas actividades, nuevos productos o de incorporar más fases del proceso productivo. La función empleadora de las cooperativas y sociedades laborales incide así mismo en otro de los objetivos del desarrollo, el de fijar la población al medio rural reduciendo así el despoblamiento.

Tal y como señalaban diferentes aportaciones, las empresas objeto de este estudio facilitan la inserción laboral de grupos desfavorecidos como por ejemplo las mujeres, para las cuales las cooperativas proporcionan interesantes posibilidades que se apoyan en: la flexibilidad de horarios que permite la conciliación de las responsabilidades familiares con las profesionales; las oportunidades para las mujeres en la medida en que la asunción de responsabilidades y competencias las capacita de cara a su posible reconversión hacia otros sectores.

El papel de las entidades en los procesos de desarrollo de sus entornos se evidencia no sólo por las intensas relaciones establecidas entre aquéllas y éstos, sino en la actitud comprometida de las personas al frente.

Por su eficacia en estas cuestiones, las empresas proporcionan el marco idóneo de aplicación para la planificación pública, prueba de ello lo constituye el amplio reconocimiento de los poderes públicos hacia la función de la Economía Social en el desarrollo, tanto en la teoría como en el diseño y aplicación de medidas de apoyo.

\section{Referencias bibliográficas}

BuEndía MARTíneZ, I. (1999). La integración comercial de las sociedades cooperativas. Consejo Económico y Social. Madrid.

CARrillo Benito, E. (1999). Guía básica de empleos de futuro en Andalucía. Sevilla.

Ceña Delgado, F. (1993). Planteamientos teóricos del desarrollo rural. Revista de Estudios Agrosociales, 169: 11-52.

DiARIO OfiCIAL L 207 de 18.8.2003. Reglamento (CE) n 1435/2003 de 22 de julio de 2003.

DiARIO OFICIAL L 207 de 18.8.2003. Directiva 2003/72/CE del Consejo, de 22 julio de 2003.

EtXeZArreta Zubizarreta, M. (1988). Concepto y objetivos del desarrollo rural integrado. En: Desarrollo rural integrado. MAPA. Madrid.

GARCía MARCOS, C. (1996). Economía Social y autoempleo colectivo. En: I Feria de Empresarias y Emprendedoras de Málaga, 25-30 de noviembre, 1996. Málaga.

GARCía MARCos, C. (1999). Las mujeres y la Economía Social. En: Las mujeres construimos Europa. Congreso Internacional sobre el empleo, 28, 29 y 30 de octubre, 1998. Ministerio de Trabajo y Asuntos Sociales. Madrid. 
Larrubia VArgas, R., NAVArro Rodríguez, S. y Nieto Figueras, C. (2004). La evolución de la política europea en el medio rural. Las estrategias LEADER en la provincia de Málaga. Universidad de Málaga. 85 p. En prensa.

NAVARRO, S. y LARRUBiA, R. (2001). Los programas Leader II en la provincia de Málaga. Su contribución al desarrollo del espacio rural. Baética, 22: 109-145.

Nieto Figueras, C. (2001). Economía Social y desarrollo rural. Baética, 23: 147-169.

Nieto Figueras, C. (2003). Mujeres empresarias y desarrollo rural. Experiencias en los municipios malagueños. Universidad de Málaga. Málaga.

OCAÑA OCAÑA, C. (1995). La población y el desarrollo rural. Una visión andaluza. En: Hacia un nuevo sistema rural (Ramos, E. y Cruz Villalón, J., Coord.). MAPA.

Rego VeigA, G. (1992). Administraciones locales y desarrollo endógeno. Estudios Territoriales, 38: 103-114.

VAlCÁRCEL-Resalt, G. (1999). Bases del Desarrollo Local sustentable. En: Manual de Desarrollo Local (Rodríguez, F., Ed.). Gijón.

VÁZQUEZ BARQUERO, A. (1987). Áreas rurales con capacidad de desarrollo endógeno. Madrid.

VÁZquez BARQuero, A. (1988). Desarrollo Local. Una estrategia de creación de empleo. Madrid.

VV. AA. (2000). Economía Social y empleo en la Unión Europea. CIRIEC. Valencia. 
